Thomas Gross ${ }^{1}$

D

'Dept of Internal Medicine, Carver College of Medicine and the University of lowa, lowa City, IA, USA.

${ }^{2}$ Dept of Pathology, Carver College of Medicine and the University of lowa, lowa City, IA, USA.

\title{
A young man with dyspnoea and audible expiration: the loops never lie
}

\section{Case report}

A 28-year-old Asian man noted the insidious onset of exertional dyspnoea over a 4-month period. This was associated with an intermittent dry cough and expiratory wheezing noted only with exercise. He noted no specific aggravating or alleviating factors and denied associated haemoptysis, chest pains, acid reflux, or systemic findings such as fever, sweats or weight loss. He had no prior history of childhood asthma. He was a fulltime student without a recent change in environmental or occupational exposures. He denied choking while eating or drinking, changes in his voice or exposure to ill contacts. Evaluation by his primary care provider included a normal physical examination and a normal chest radiograph (figure 1). Spirometry was interpreted as showing a moderately severe obstructive defect (figure 2; initial presentation). There was no reversibility with bronchodilator (not shown).

\section{Task 1}

What would be your first intervention?

a. Spirometry pre- and post-exercise challenge

b. Computed tomography of the chest looking for a parenchymal lung disease

c. Trial of asthma controller therapy

d. Exercise reconditioning programme

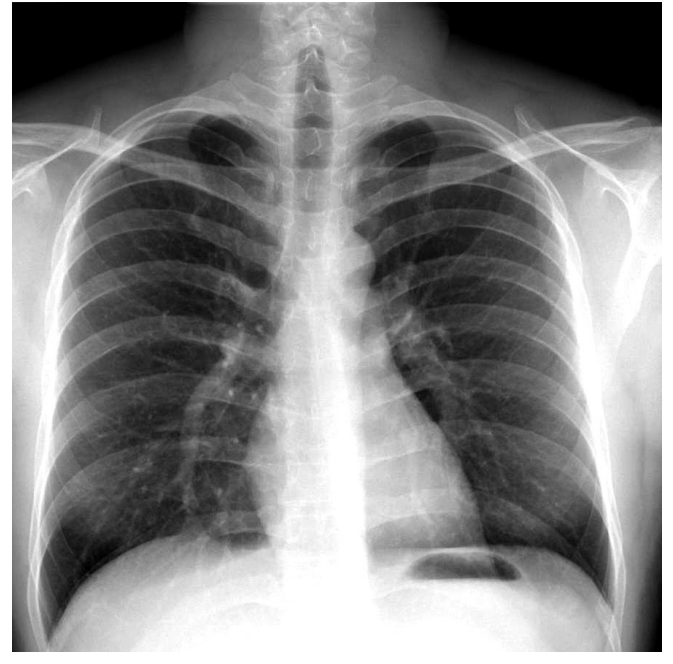

Figure 1 Chest radiograph at the time of initial presentation with cough and audible breath sounds. Note the lack of obvious volume loss or visible lesion.
Cite as: Taher $\mathrm{H}$, Samuelson $\mathrm{M}$, Gehlbach B, et al. A young man with dyspnoea and audible expiration: the loops never lie. Breathe 2016; 12: 159-163.

@ERSpublications

Spirometry provides clues to solving this puzzle of dyspnoea and wheeze in a young nonsmokerthe loops never lie! http://ow.ly/YC9z| 


\begin{tabular}{lcccc}
\hline & Initial presentation & Follow-up visit & $\begin{array}{c}\text { After follow-up } \\
\text { visit }\end{array}$ & $\begin{array}{c}\text { Following final } \\
\text { treatment }\end{array}$ \\
\hline FVC L & 3.88 & 3.82 & 2.45 & 4.19 \\
FVC \% pred & 77 & 75 & 48 & 83 \\
FEV 1 L & 2.29 & 1.97 & 1.92 & 3.21 \\
FEV $_{1} \%$ pred & 55 & 47 & 46 & 77 \\
FEV $_{1}$ /FVC & 0.59 & 0.52 & 0.79 & 0.77
\end{tabular}

Flow-volume loop
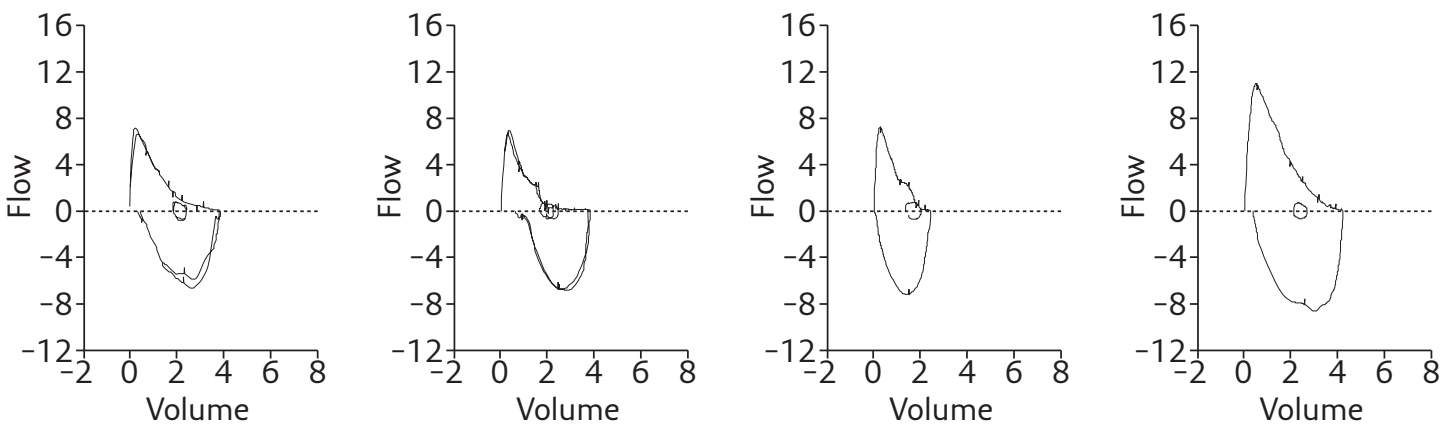

Figure 2 Serial measurement of lung function via spirometry with accompanying flow-volume loops.

\section{Answer 1 \\ c. Trial of asthma controller therapy}

The aetiology of exertional shortness of breath in otherwise healthy young adults is most commonly due to disorders such as asthma, deconditioning, anxiety or vocal cord dysfunction. Spirometry reveals a clearly obstructive ventilatory defect, with the reduction in forced expiratory volume in $1 \mathrm{~s}$ (FEV1) exceeding the change in forced vital capacity (FVC) leading to a reduced FEV1/FVC ratio $[1,2]$. The lack of response to a single dose of a short-acting bronchodilator does not exclude the possibility of asthma [2]. This may be particularly true in the setting of chronically undertreated disease associated with airway oedema and remodelling. Although the reduction in FVC is not characteristic of pure airways obstruction, the normal chest radiograph and lack of crackles on examination make occult parenchymal disease in this young nonsmoker less likely. The combination of obstructive spirometry and expiratory noise led his physicians to initially suspect bronchospastic lung disease. Exercise typically exacerbates patients with asthma and the utility of an exercise challenge lies in uncovering occult asthma in subjects with normal baseline spirometry. He was started on an inhaled corticosteroid and a short-acting bronchodilator to use prior to exercise.

After a 4-week empiric clinical trial, the subject noted no symptomatic improvement and spirometry values were unchanged. In fact, he now noted expiratory sounds at lower levels of exertion and even with forced expiration at rest. He was referred to the pulmonary specialty clinic.

Examination in the pulmonary specialty clinic revealed a young man with a healthy appearance in no respiratory distress at rest or with walking down the hall. His voice was normal. There was no palpable goitre and no cervical adenopathy. Lung examination revealed mild prolongation of the expiratory phase with a coarse wheeze heard best at the base of the neck anteriorly. It was accentuated with forced expiration and could occasionally be elicited with brisk inspiration. Examination by several physicians confirmed these findings and the use of an electronically amplified, dual headed stethoscope did not lateralise the sounds. Spirometry data was reviewed in more detail (figure 2; follow-up visit).

\section{Task 2}

Based on this data review, which of the following would you choose?

a. Increase potency of asthma treatment, as airways obstruction is worse.

b. Laryngoscopy to look for structural or functional upper airway abnormalities.

c. Anxiolytic medications and speech therapy for laryngeal dyskinesia

d. Airway reconstruction CT imaging to look for tracheomalacia. 


\section{Answer 2}

b. Laryngoscopy to look for structural or functional upper airway abnormalities.

Although the decrease in early expired volumes does imply worsening obstruction, we felt the lack of a clinical response to appropriate therapy and the physical findings did not support ongoing concern for bronchial asthma. The typical findings for vocal cord dysfunction are a flattening or attenuation of the inspiratory limb, the so-called variable extrathoracic upper airways obstruction, associated with inspiratory stridor [2, 3]. However, there are case reports of expiratory sounds and obstruction due to inappropriate partial glottic closure, particularly with stress or exertion [4, 5]. Anxiety may contribute to vocal cord dysfunction and speech therapy interventions are invaluable in the treatment; we felt the progression of this patient's complaints warranted a less empirical approach. Mid-to-late expiratory airway collapse with wheeze is commonly seen in patients with tracheobronchomalacia [6]. However, this subject had no history of an event that could have precipitated airway injury, was not obese and his symptoms seemed to progress more rapidly than would be typical for this more chronic condition. We were left contemplating either a vocal cord problem with inappropriate expiratory glottic closure or a progressive variable intrathoracic obstruction. Given the normal chest radiograph and the nonlateralising examination, we proceeded to laryngoscopy during exercise on a bicycle.

The subject was seated on an exercise bicycle with full 12-lead ECG monitoring and a forehead oxygen saturation probe. A flexible rhinoscope was passed via the right nare after topical anaesthesia with a water-based lubricant containing $2 \%$ lidocaine. Laryngoscopy showed normal upper airway anatomy without oedema and vocal cords that moved normally with voluntary phonation. The subject then pedalled the bike at increasing workload until the expiratory sound was audible. The operator documented a mid-range pitched expiratory wheeze emanating from the airway without apparent glottic closure or abnormal collapse of the upper airway. A limited view of the proximal trachea without crossing the vocal cords showed no apparent obstruction or dynamic collapse (see the supplementary material for a 10 s recording of expiratory sounds).

Over the next few weeks, the patient noted persistent dyspnoea and a nonproductive cough with less expiratory noise. Spirometry was repeated (figure 2; after follow-up visit).

\section{Task 3}

Based on this new data review, which of the following would you choose?

a. Spirometry variability implies variable effort. The patient needs counselling and anxiolytics.

b. There is now a restrictive defect. Evaluate for respiratory muscle strength.

c. There is now a restrictive defect. Carry out a CT scan to look at lung parenchyma and pleura.

d. Bronchoscopy to evaluate for progressive intrathoracic airway obstruction. 


\section{Answer 3}

d. Bronchoscopy to evaluate for progressive intrathoracic airway obstruction.

It is true that a variable performance on spirometry testing can be a clue indicating underlying malingering or variable tone in the larynx with vocal cord dysfunction [2]. However, on each day of testing, our subject exhibited highly reproducible testing giving credence to the veracity of the data produced. The spirometry now clearly shows a restrictive ventilatory defect with a symmetric reduction in $\mathrm{FEV}_{1}$ and FVC leading to a preserved FEV1/FVC ratio. Changes in lung parenchymal compliance, chest wall elastance, weakness or paralysis of respiratory muscles, and impingement by effusions, ascites or central obesity may all superimpose a restrictive ventilatory defect. However, this subject's history was more supportive of an airway issue. We felt that the evolution of flow-volume loops represented the progression of a large airway obstruction. ACT scan, in retrospect, would have led to the diagnosis, but we chose instead to proceed directly to bronchoscopy to examine the central airways.

At bronchoscopy, we encountered a mass occluding the left mainstem bronchus (figure 3). It was possible to push past the lesion and all segmental airways were identified. The lesion was pedunculated and arose from the membranous surface of the main airway. The tumour was debulked using an electrocautery snare and forceps with minimal residual disease. Histopathology revealed an outer layer of benign airway epithelium with an underlying infiltrating tumour composed of a mixed population of cells: cuboidal cells manifesting well-formed glands with copious mucin, some with a squamous differentiation, and others with an intermediate phenotype (figure 4). There was no necrosis and only very rare mitotic

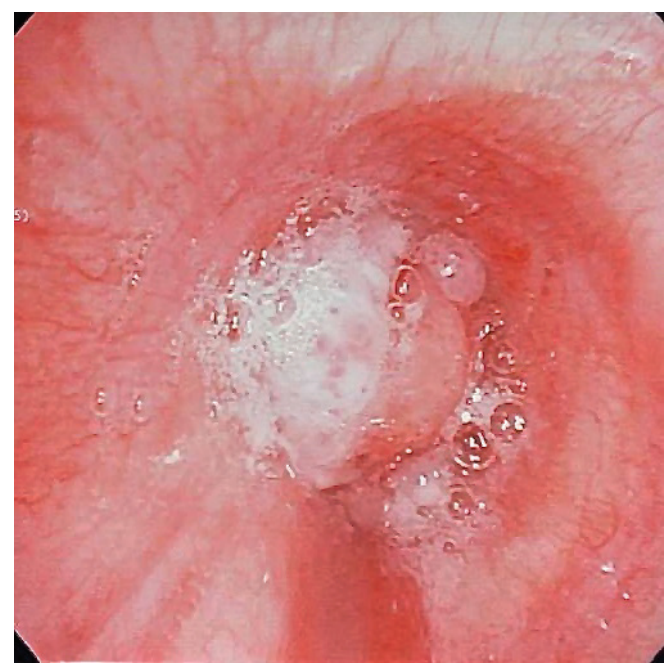

Figure 3 Bronchoscopic view of left mainstem bronchus showing a polypoid mass with subtotal airway obstruction (bubbles reflect expiratory air flow).

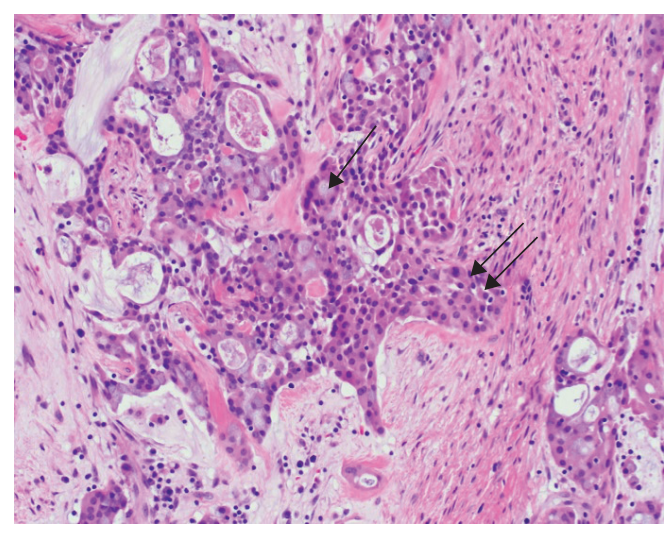

Figure 4 Histopathology of the endobronchial mass following bronchoscopic resection. At 200x magnification, note the infiltrating population of tumour cells with varying phenotypes including mucin-secreting cells (single arrow) and others with more squamoid morphology (double arrow).

figures. This pattern was felt most evocative of a salivary gland neoplasm with features of a low-grade mucoepidermoid carcinoma (MEC). Immunohistochemistry staining revealed the tumour did not express thyroid transcription factor-1. Fluorescent in situ hybridisation analysis for the MECT1-MAML2 fusion protein was positive in $92 \%$ of the malignant cells, consistent with a lowgrade mucoepidermoid neoplasm [7]. A positron emission tomography (PET) CT scan revealed no evidence of extrabronchial, mediastinal, or parenchymal disease; there was no abnormal 2-fluoro2-deoxy-D-glucose (FDG) uptake anywhere. Repeat airway examination with biopsies at the prior excision site revealed only granulation tissue without residual tumour. Repeat spirometry showed near normalisation of expired volumes with a normal ratio and flow-volume loop (figure 2; following final treatment).

\section{Discussion}

This young man presented with progressive dyspnoea and abnormal pulmonary function testing. The aetiology of exertional shortness of breath in otherwise healthy young adults is most commonly due to disorders such as asthma, deconditioning, anxiety or vocal cord dysfunction; an endobronchial neoplasm is low on the list. The combination of obstructive spirometry and expiratory noise led physicians to initially treat the patient for bronchospastic lung disease. Physical examination by several seasoned clinicians described mid-range pitched expiratory wheezes best heard in the upper chest and neck with only rare abnormal inspiratory sounds. Examination with an electronic dual headed stethoscope did not lateralise the sounds. Even at the time of exercise laryngoscopy, the sound was noted to be loudest in the throat, but was solely expiratory in phase. Inspiratory stridor is classically taught to emanate 
from the supraglottic larynx. Expiratory stridor should direct diagnostic evaluation to the tracheobronchial tree for intrathoracic obstruction.

Spirometry is essential to the diagnosis and management of obstructive lung disease. Although professional organisations differ on exact definitions, all agree that obstruction is characterised by impaired exhalation manifest as a reduction in the $\mathrm{FEV}_{1} / \mathrm{FVC}$ ratio below the lower limit of normal with the severity of the obstruction reflected in the degree of reduction in the $\mathrm{FEV}_{1}[1,2]$. In this case, the initial spirometry revealed a clear reduction in $\mathrm{FEV}_{1} / \mathrm{FVC}$ and $F E V 1$ with comparative preservation of the FVC. This is consistent with moderate to severe airways obstruction and was reflected in the flow-volume loop with coving of the expiratory limb (figure 2; initial presentation). Repeat testing 6 weeks later showed a worsening obstructive defect. There was further coving of the terminal expiratory limb. This was interpreted as possible early partial glottic closure and vocal cord dysfunction was thought a plausible explanation for the variability in loops and expiratory sounds apparently emanating from the upper airway. In retrospect, careful examination of the flow-volume loops reveal a more "biphasic" pattern with normal initial phases to both the expiratory and inspiratory limbs, followed by terminal flattening (figure 2; follow-up visit). This pattern has been described in single lung transplant patients and may herald bronchial anastomotic stenosis [2, 8, 9]. Finally, despite an improvement in expiratory noise, dyspnoea persisted and spirometry revealed a considerable reduction in the FVC, with normalisation of both the $\mathrm{FEV}_{1} / \mathrm{FVC}$ and flowvolume loop suggesting a new superimposed ventilatory restriction (figure 2; at the time of bronchoscopy). This was due to complete obstruction of the left mainstem bronchus and, functionally, a pneumonectomy (figure 3). The evolution of the flow-volume loop nicely demonstrates the airflow physiology of progressive large airway obstruction. The contour of the flow-volume loop contains valuable information and a change over time may accurately reflect evolving pathophysiology.

Progressively obstructing central airway tumours in young patients are rare; retained foreign bodies with granulation tissue, hamartoma, eroding broncholith, granulomatous infection and well-differentiated neuroendocrine tumours (carcinoid) head up differential diagnostic lists. The incidence of malignant tumours is much lower than in older smokers where bronchogenic carcinoma would be the most common aetiology. Endobronchial biopsy in this case revealed a low grade MEC.

The rare occurrence of primary salivary gland tumours in the airway makes optimal treatment decisions a challenge. MEC is an uncommon tumour without clear risk factors and presents in a younger age group than typical bronchogenic carcinoma [10, 11]. In the case of low grade MEC, tumour recurrence after excision has been only rarely reported, making surgical options attractive and potentially curative. Long-term follow-up data suggests 5 -and 10-year survival rates that exceed 10 years in the absence of documented regional spread [10]. The use of PET scans in a few cases has demonstrated FDG uptake in both the primary tumour and metastatic deposits offering hope this may prove useful as a screening modality in long-term surveillance [11]. Given the absence of detectable residual disease and the paucity of data to guide adjuvant treatments, this patient is contemplating options of pneumonectomy versus close surveillance.

This article has supplementary material available from breathe.ersjournals.com

\section{Conflict of interest}

None declared.

\section{References}

1. Global Initiative for Chronic Obstructive Lung Disease (GOLD). Global Strategy for Diagnosis, Management and Prevention of COPD 2016. www.goldcopd.org/ Date last updated: December 2015. Date last accessed: March 17, 2016

2. Pellegrino R, Viegi G, Brusasco V, et al. Interpretative strategies for lung function tests. Eur Respir J 2005; 26: 948-968.

3. Newman KB, Mason UG 3rd, Schmaling KB. Clinical features of vocal cord dysfunction. Am J Respir Crit Care Med 1995; 152: 1382-1386.

4. Goldman J, Muers M. Vocal cord dysfunction and wheezing. Thorax 1991; 46: 401-404

5. Bahrainwala AH, Simon MR, Harrison DD, et al. Atypical expiratory flow volume curve in an asthmatic patient with vocal cord dysfunction. Ann Allergy Asthma Immunol 2001; 86: 439-443.

6. Carden KA, Boiselle PM, Waltz DA, et al. Tracheomalacia and tracheobronchomalacia in children and adults: an in-depth review. Chest 2005; 127: 984-1005.
7. Seethela RR, Dacic S, Cieply K, et al. Reappraisal of the MECT1/MAML2 translocation in salivary mucoepidermoid carcinoma. Am J Surg Pathol 2010; 34: 1106-1121.

8. Ross DJ, Belman MJ, Mohsenifar Z, et al. Obstructive flowvolume loop contours after single lung transplantation. J Heart Lung Transplant 1994; 13: 508-513.

9. Anzueto A, Levine S, Tillis W, et al. Use of the flow-volume loop in the diagnosis of bronchial stenosis after single lung transplant. Chest 1994; 105: 934-936.

10. Liu X, Adams A. Mucoepidermoid carcinoma of the bronchus: a review. Arch Pathol Lab Med 2007; 131: 14001404.

11. Li X, Zhang W, Wu X, et al. Mucoepidermoid carcinoma of the lung: common findings and unusual appearances on CT. Clin Imaging 2012; 36: 8-13. 